\title{
Stato e mercato tra storia ed economia: la questione migratoria
}

\author{
di Lea Ypi
}

Feb 9, 2021 | Emergenza e (nuova) normalità, In evidenza, Stato/Mercato | $\underline{0}$ |

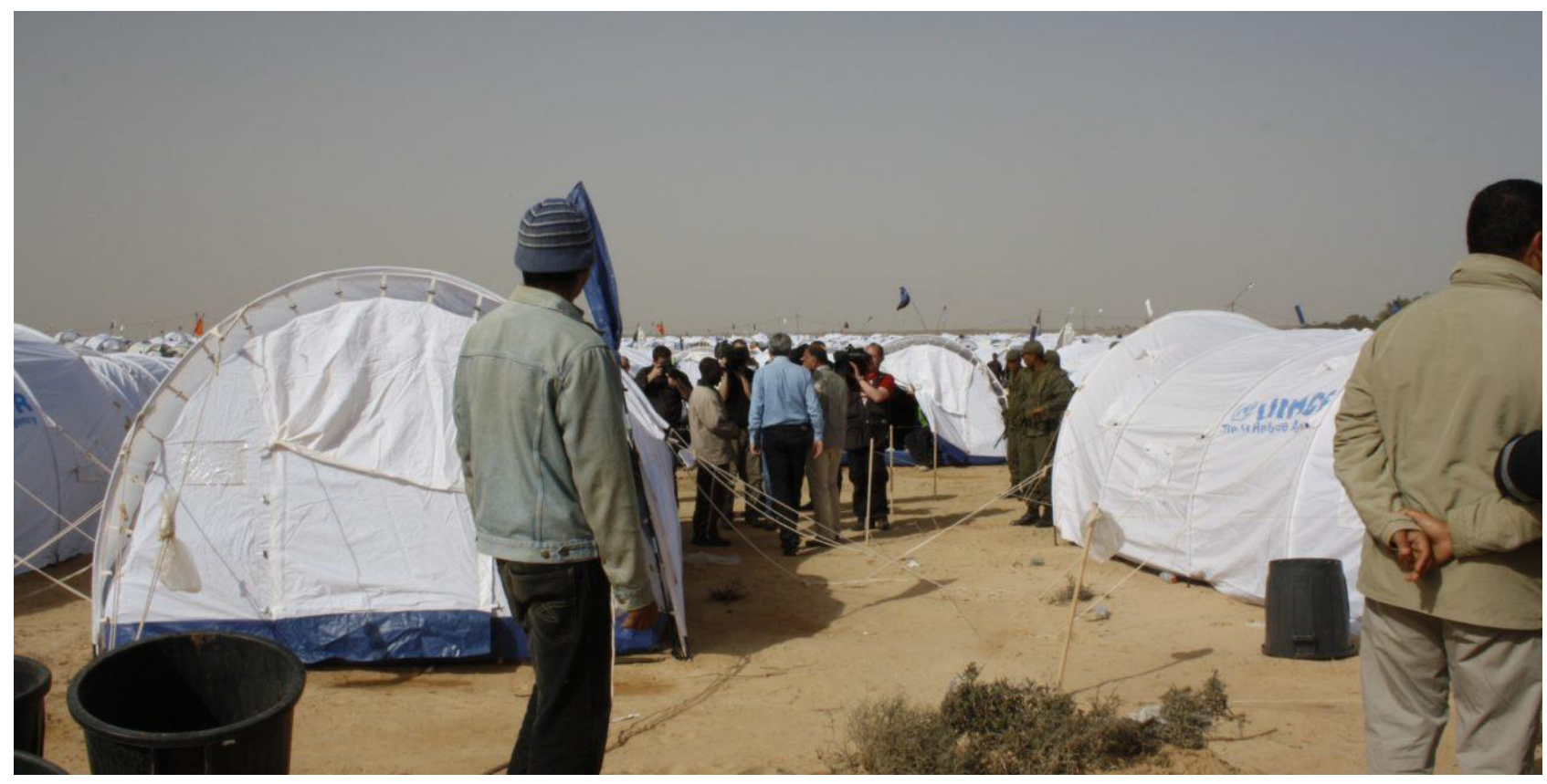

Vista di un campo di transito vicino al confine tunisino con la Libia.

By DFID - UK Department for International Development - Flickr: Transit camp for migrants near the Tunisian border with Libya, CC BY 2.0, Link

\section{Il dossier sulla Summer school 2020 si compone di 5 coppie di parole chiave. Questo testo afferisce alla coppia di parole chiave Stato/Mercato}

\begin{abstract}
Nella terza giornata della Summer School 2020 abbiamo chiesto a Emanuele Felice e Lea Ypi di riflettere con noi sulle parole chiave Stato e Mercato.

Secondo una certa narrazione delle destre europee, che fa breccia anche nel mondo progressista, la presenza dei migranti sul mercato del lavoro sarebbe responsabile della perdita di forza contrattuale della classe lavoratrice locale. Si tratta di una narrazione che si serve dell'immigrazione come facile scorciatoia per interpretare i conflitti di classe che caratterizzano il nostro tempo. I governi, nella difficoltà di gestire i mutamenti portati dalla globalizzazione, pare quasi cerchino una strada di autoassoluzione. In tale contesto anche la cittadinanza si modifica, trasformandosi da bene politico in una sorta di diritto di nascita. Peraltro, chi dispone di risorse economiche può spesso acquistarla: la cittadinanza diviene così una merce come tutte le altre. Compito precipuo e urgente, oggi, è iniziare a considerare diversamente il problema della migrazione nel suo complesso, mettendo in discussione i termini con cui viene evocato quotidianamente. E procedere a un rinnovamento dei
\end{abstract}


partiti e dell'azione politica, in modo da ristabilire un legame con le masse senza diritti a livello globale.

\section{Rifiutare una falsa narrazione}

Secondo una certa narrazione, piuttosto invasiva oggi anche presso l'opinione pubblica di stampo progressista, la presenza dei migranti sul mercato del lavoro sarebbe responsabile della perdita progressiva di forza contrattuale, e anche di tutele, subita dalla classe lavoratrice.

Si tratta di una narrazione infondata che occorre rifiutare e, per farlo, è necessario prima di tutto procedere a un'analisi empirica del problema migratorio.

Da un lato, i dati dei flussi ci dicono che, malgrado il numero delle persone che vivono fuori dal loro paese di nascita sia cresciuto in termini assoluti, si tratta di un numero in linea con la crescita della popolazione globale. In proporzione ai flussi del passato, insomma, non è cambiato niente.

Dall'altro lato, se si pensa al contributo dei migranti nelle società ospiti, non ci sono davvero prove che i migranti siano un onere in termini assoluti. Eppure, nonostante questo, nel discorso politico ci si riferisce ugualmente alla migrazione come a un problema.

In effetti lo è ma non da un punto di vista demografico e neanche culturale: è un problema politico, che poco ha a che fare con i migranti ma piuttosto con il modo in cui la migrazione viene gestita dalle classi dirigenti, come peraltro è già accaduto in passato. La storia insegna che le élites al potere si sono rifiutate di garantire i diritti alla parte vulnerabile della società, salvo poi accusarla di mancata integrazione e trasformarla in bersaglio agli occhi del resto della popolazione. La divisione che oggi viene sottolineata tra autoctoni e stranieri -e che è centrale al dibattito sulla migrazione- altro non è se non la riproposizione di uno schema di esclusione già adottato in passato.

Peraltro, il problema non sembrano essere i migranti in sé, ma la classe sociale cui appartengono. Si pensi al fatto che i migranti benestanti sono i benvenuti; l'acquisizione della cittadinanza, infatti, non è mai stata così facile per i ricchi, per gli investitori, per gli imprenditori, per i professionisti. Ad essere invece sistematicamente esclusi e privati di diritti sono i migranti poveri: gli irregolari che sono costretti in centri di detenzione, che vogliono raggiungere le loro famiglie ma non guadagnano abbastanza o non hanno un livello di istruzione sufficiente a superare i test di cittadinanza, e così via.

Se ci soffermiamo su questo dato possiamo senz'altro affermare che gli stati liberali occidentali stanno fallendo su molti fronti: da una parte, c'è un'incapacità dei governi a rispondere alle esigenze dei ceti più bisognosi (cittadini e non cittadini), a gestire un'economia che funzioni per tutti; dall'altra, c'è un indebolimento della capacità di intervento politico da parte dei movimenti progressisti.

Di fronte a queste due questioni, l'immigrazione offre semplicemente un "modo spiccio" per interpretare i conflitti cui la società si trova di fronte. La destra coglie l'esistenza di tali conflitti, ma non le cause. La comunità politica, per parte sua, accusa dei propri fallimenti quelli che non ne fanno parte, evitando in questo modo di farsi carico delle responsabilità dei propri, di fallimenti.

Il problema di fondo, invece, ha a che fare con la trasformazione della cittadinanza, che negli ultimi decenni da bene politico -definito dalle forze progressiste come un processo di apertura degli spazi pubblici democratici- si è ridotta principalmente a una questione di diritto di nascita, che si ottiene attraverso l'appartenenza etnica nazionale. Da una parte, quindi, la cittadinanza come spazio 
progressista di inclusione e di apertura di diritti, e dall'altra la cittadinanza come un diritto quasi ereditario, simile allo status nel Medioevo, che si acquisisce a partire dalla nascita in un determinato Paese, o che si deve guadagnare con altri mezzi, finanziari o di altro tipo.

Se pensiamo alle modalità con cui la cittadinanza viene estesa ai membri potenziali della società, una delle più significative è senza dubbio la capacità di acquistare tale la cittadinanza, $\mathrm{o}$ anche un permesso di soggiorno accelerato, da parte di chi dimostra di avere i mezzi per dare un contributo produttivo al Paese accogliente. Questo significa che gli investitori finanziari, cioè coloro che sono disposti a pagare molto di più per avere un passaporto che dia diritto a benefici, hanno un vantaggio rispetto a chi, invece, non ha le risorse, la possibilità di fare la stessa offerta. In questo senso si può dire che la cittadinanza diviene un bene di mercato, che può essere venduto e comprato come altri beni. Se questo è vero può accadere che, allorquando tale scambio non può avvenire per mancanza di mezzi economici, il potenziale cittadino si trova costretto a dimostrare di meritarsi l'accesso alla comunità politica, a questo "privilegio" che è la cittadinanza di un Paese a capitalismo avanzato, mediante strumenti (test linguistici, sulle competenze ecc.) che si applicano a residenti a lungo termine.

Intendere la cittadinanza in questo modo significa rafforzare il carattere di classe dello Stato. E dire che lo Stato ha un carattere di classe equivale a dire che la capacità dello Stato di agire come piattaforma politica attraverso la quale si risolvono i conflitti fra gruppi sociali in modo equilibrato e mediante lo scambio democratico, viene progressivamente erosa. Lo Stato diventa allora lo strumento che serve gli interessi di potenti élite, sia politiche che finanziarie.

Quando la cittadinanza viene acquistata e venduta, invece che essere veicolo di emancipazione diventa uno strumento di dominio e di oppressione. La democrazia si trasforma in una forma di oligarchia, attraverso la quale una minoranza ricca e di maggior potere, politico e finanziario, si appropria dei mezzi attraverso i quali si può acquisire e gestire quello stesso potere.

Il modello di cittadinanza come diritto ereditario si contrappone a un modello di cittadinanza che di cui stiamo perdendo le tracce, che potremmo definire democratico, universale e progressista, secondo il quale le minoranze escluse vengono a far parte della sfera pubblica grazie alla partecipazione attiva e vengono finalmente riconosciute come parte della comunità politica.

Quando invece la cittadinanza si trasforma in una merce come tutte le altre, lo Stato, anziché aiutare a domare il potere del mercato, si arrende completamente a questo potere dell'economia con tutte le ripercussioni che ne derivano: l'ascesa delle destre, la strumentalizzazione del problema dell'immigrazione.

\section{Ripensare la politica delle sinistre sulla migrazione}

Se la situazione reale è quella in cui la cittadinanza diventa una merce di scambio e lo Stato abdica al suo ruolo, quale risposta politica dovrebbero dare le sinistre?

Anzitutto, si dovrebbero domandare se non sia opportuno considerare diversamente la migrazione, ovvero non tanto come fonte di problemi per la sinistra, ma come sintomo di una sua crisi. Che andrebbe superata.

Una proposta di sinistra dovrebbe, infatti, cominciare con il mettere in discussione i termini con cui la migrazione viene evocata quotidianamente, nel discorso politico, dalla destra. 
In passato, la sinistra è stata internazionalista ed orientata, almeno in via di principio, a rispondere alle esigenze dei lavoratori in tutto il mondo, indipendentemente dalla razza, dalla religione, dal genere o dall'etnia. Il vecchio slogan "proletari di tutto il mondo unitevi" significava qualcosa solo quando chi lo pronunciava credeva che i conflitti politici potessero essere articolati secondo linee di classe e affrontati in quanto tali nel dibattito pubblico. È quindi importante realizzare che l'aver abbandonato questa prospettiva rappresenta una delle perdite più importanti, negli ultimi decenni, per le forze progressiste.

Oggi l'emancipazione è vista, dalla sinistra, come una questione che si connette alle leggi e ai diritti, una questione che riguarda chi fa e promulga le leggi, chi è incluso e chi è escluso. La conseguenza è che il problema delle migrazioni è affrontato come se fosse quello di regolare l'appartenenza politica, non di conferire potere alle classi sfruttate, per le quali i confini dell'oppressone non coincidono mai esattamente con quelli dello Stato nazionale. In questo senso l'appartenenza politica è ormai vista dalla sinistra come "la" soluzione. Se, però, non si mette in discussione l'intero modo di articolare il problema, se non si difendono i migranti vulnerabili senza se e senza ma, se non si spiega che il problema non è la diversità culturale ma l'ingiustizia di classe, è facile comprendere come qualunque soluzione proposta finisca per portare all'esclusione e, quindi, per giocare a favore delle destre.

E del resto non è difficile spiegare perché la sinistra è passata dall'essere un progetto incentrato sull'emancipazione di una classe sociale a essere un progetto incentrato sulla difesa dello Stato democratico.

Alla fine della guerra fredda, questa compagine politica si è liberata dall'associazione con il socialismo di stato e ha pensato di salutare l'inizio di un nuovo ordine mondiale improntato alle opportunità, il benessere, la democrazia, il rule of law, l'impegno globale per i diritti umani. All'inizio degli anni Novanta e con questi nuovi slogan, la sinistra, il centrosinistra, il new labour, i socialdemocratici se la sono cavata abbastanza bene dal punto di vista elettorale; sono stati in grado di influenzare l'agenda del post '89 (compresa l'agenda dell'integrazione europea) in maniera abbastanza significativa. In quegli anni è avvenuto un passaggio da un progetto (internazionalista) di emancipazione sociale attraverso il conflitto di classe ad un progetto (civiconazionale) di emancipazione politica attraverso lo stato di diritto. In questo processo, in parte dovuto anche alle dinamiche della globalizzazione, la sinistra ha abbandonato la politica (intesa come conflitto tra gruppi sociali differenti) e si è dedicata all'amministrazione (cioè al governo responsabile, che mira a condividere i benefici del libero mercato).

La questione oggi evidente, però, è che il libero mercato non ha fornito le prestazioni che ci si aspettava: sappiamo che le disuguaglianze sociali, in Europa, sono cresciute; le differenze regionali sono rimaste significative; le crisi finanziarie (e ora anche la crisi pandemica) hanno continuato a erodere il welfare state. Anche a livello internazionale, i governi di sinistra sono stati complici di diverse guerre combattute in nome della protezione dei diritti umani, che hanno portato alla distruzione, al caos (nei Balcani, nel Medio Oriente) e alla crescita del flusso dei rifugiati da quelle regioni. Il progetto di coniugare democrazia e capitalismo attraverso il miglioramento del welfare, come speravano i fautori della "terza via", è stato messo in crisi e, ormai, nessuno più dubita dell'esistenza di una crisi.

Questo stato di cose, però, solleva dubbi anche sulla possibilità di riformare istituzioni sopranazionali come l'UE, se ciò significa lasciare invariato il suo nucleo economico come è riflesso nella struttura presente nei trattati (si pensi, ad esempio, a quanto la popolarità della Brexit in Gran Bretagna sia un'espressione dell'ostilità verso le cassi dirigenti sia nazionali che europee, 
che va presa assolutamente sul serio benché in questi ultimi mesi sia cresciuta, nelle opinioni pubbliche, la fiducia verso queste stesse élite per la gestione della pandemia).

La sfida più importante per la sinistra, oggi, è, da una parte, quella di avere l'umiltà di porsi nuovamente di fronte alle esigenze di classi sociali che ha trascurato per molto tempo; dall'altra, quella di mostrarsi capace di guidare, di dare una visione di orientamento democratico a questi gruppi in modo che possano esprimere le loro esigenze senza che vengano semplicemente intercettate dalle destre (la cui ascesa fa perno sui bisogni di un elettorato che si è spostato a destra perché ha perso tutto nel processo di globalizzazione degli ultimi decenni).

L'auspicio della socialdemocrazia classica (da Bernstein in poi) è stato quello di utilizzare la democrazia per giungere all'abolizione di un governo di classe e portare i cittadini, attraverso il diritto di voto, a sentirsi soci di un'impresa cooperativa che consentisse uno sviluppo della comunità politica. Ma il presupposto di una prospettiva così ottimistica era un ideale di inclusione, di una cittadinanza potenzialmente aperta a tutti (l'ideale di cittadinanza di cui ho parlato all'inizio). Quando la socialdemocrazia faceva queste promesse, si era all'alba di un'epoca in cui la mobilitazione politica alimentava l'idea di un'espansione della cittadinanza (golden age della cittadinanza espansiva).

A mio parere oggi le condizioni per una valutazione così ottimistica non ci sono più perché la tendenza attuale va proprio in direzione contraria: nell'era della cittadinanza restrittiva (venduta e scambiata come una merce) la lotta non può più essere mediata con mezzi politici, non può più essere contenuta nei canali standard di partecipazione politica.

C'è bisogno di rivedere il concetto di rappresentanza e il rapporto fra democrazia, capitalismo e liberalismo; di capire che cosa significa la rappresentanza politica: chi viene rappresentato e in quali luoghi politici. Perché una volta che la cittadinanza diventa merce per pochi l'ideale della democrazia è una promessa vuota.

Nella retorica progressista, in tutto il mondo, si continua a celebrare l'ideale della cittadinanza come veniva intesa precedentemente a questa fondamentale trasformazione, tacendo sulla nuova tendenza all'esclusione che riguarda l'attuale cittadinanza. Né i documenti di politica pubblica socialdemocraticané i manifesti elettorali dei partiti di centrosinistra evidenziano la preoccupazione di cercare misure correttive che possano opporsi a tale tendenza, anzi si può quasi dire che il problema non venga nemmeno percepito.

Il crollo della dimensione civica in quella che si potrebbe chiamare etnopolitica è la riduzione di un ideale universale e progressista della cittadinanza a un concetto conservatore esclusivo, che non viene contrastato perché non si mettono in discussione questioni fondamentali: come intendiamo la rappresentanza politica? Come intendiamo la cittadinanza?

I movimenti e i partiti progressisti di tutto il mondo dovrebbero iniziare a cambiare profondamente $\mathrm{i}$ contenuti, dovrebbero coordinarsi per proporre una visione più radicale della cittadinanza per ripensarne le modalità di accesso. Spesso accade invece che si limitino a campagne elettorali orientate alla vittoria elettorale su base nazionale: un risultato che - anche se ha il beneficio immediato di permettere di restare al governo - è illusoriamente trasformativo. 
Quale potrebbe essere, allora, la direzione da intraprendere per superare non soltanto la crisi migratoria (assai presente nel discorso pubblico), ma anche la crisi economica, la crisi ambientale e ora anche la crisi provocata dalla pandemia e contribuire alla costruzione di un mondo sostenibile?

Per provare a rispondere a questa domanda occorre partire da una premessa indispensabile: la questione dell'interdipendenza fra democrazia nazionale, cittadinanza e progresso a livello globale è arrivata a un momento di impasse, che ci obbliga a un cambio di visione, a una revisione del ruolo dello Stato in generale e del suo rapporto con la democrazia nazionale.

Il problema è che sia l'esclusione all'interno dello Stato che le esclusioni che avvengono fra gli stati si sostengono reciprocamente e quindi servono a consolidare un ordine economico che non viene contrastato al suo centro. Questa vicenda racconta del rapporto fra capitalismo e (presunto) Stato democratico; e se la narrazione non cambia è un problema per tutti, non solo per gli immigrati.

È difficile dire quali siano le alternative, visto che il problema è globale. Tradizionalmente, la rappresentanza ha fatto uso del partito politico come strumento per democratizzare la politica di massa. Oggi però non è più possibile ignorare la crisi della rappresentanza e quindi sarebbe utile ritornare sui nostri passi e riproporre tutte le domande fondamentali della politica. Ad esempio: che cosa significa avere una politica che si basa solo sulla policy, senza una visione di trasformazione fondamentale della società? Il trionfo del managerialismo tecnocratico. Che cosa significa avere delle visioni alternative ma senza avere degli attori collettivi che ci permettano di incanalarle? Il trionfo del personalismo in politica, l'ascesa del leader carismatico. Che cosa significa pensare l'azione collettiva solo a livello nazionale di fronte a una crisi ambientale globale? L'avanzata del populismo e il rischio del fascismo.

Nell'attuale crisi bisognerebbe pensare a come è possibile rinnovare il ruolo dei partiti in modo da ristabilire un legame con le masse senza diritti. Questo non significa semplicemente resuscitare il fantasma del partito di massa nazionale, ma cercare di capire come possiamo riadattare il concetto di partito alle attuali condizioni di globalizzazione.

Altre domande cruciali riguardano i partiti e la loro attuale utilità. In che rapporto stanno con la sovranità? Quanto è importante per i partiti che ci siano elezioni nella logica della rappresentanza liberale per come l'abbiamo intesa finora? Come possono guidarci verso una via di uscita?

Per rispondere a domande di questo genere occorre un cambiamento radicale di prospettiva. Da una parte i partiti devono guardare al loro interno e dall'altra all'esterno. A livello interno, se i partiti di sinistra riescono a ridiventare la voce dei lavoratori, dei migranti vulnerabili, dei disoccupati, delle donne sfruttate che svolgono il lavoro di cura, dei precari della gig economy, allora saranno in grado di trasformare le proteste sociali isolate in richieste politiche di portata universale. A livello internazionale, i partiti di sinistra saranno in grado di trasformare la società solo se sapranno anche impegnarsi per la trasformazione dell'ambiente e se saranno in grado di andare oltre i confini nazionali, costruendo reti di cooperazione internazionale. Si tratta, anche per i partiti, di reinventare se stessi esplorando forme di attivismo, di mobilitazione, di deliberazione democratica che siano in grado di usare la partecipazione nell' era digitale, rispondendo alle sue sfide.

Tutto ciò significa superare la tradizionale concezione dei partiti come macchine elettorali, il cui scopo principale è vincere le elezioni per formare maggioranze in parlamento.

Si tratta di recuperare un modello di partito che -come diceva Gramsci- funga da moderno Principe: promotore di visione di cambiamento sociale come movimento, come momento fondante di un tipo diverso di società, di politica e di rapporti economici. 
